\title{
Engineering Lifetime Model of Crosslinked Polyethylene under Electrical and Thermal Stresses
}

\author{
Sarawuth Pramualsingha and Peerawut Yutthagowith* \\ School of Engineering, King Mongkut's Institute of Technology Ladkrabang, \\ 1 Soi Chalongkrung 1, Ladkrabang, Bangkok 10520, Thailand \\ (Received January 29, 2021; accepted June 14, 2021)
}

Keywords: electrical and thermal stresses, engineering model, lifetime estimation, power cable, XLPE material

This paper presents an effective engineering lifetime model of crosslinked polyethylene (XLPE), which was subjected to electrical field stresses in the range of 7 to $90 \mathrm{kV} / \mathrm{mm}$ and temperatures of 20 to $90{ }^{\circ} \mathrm{C}$. The inversed power model (IPM) is widely used for estimating the lifetime of XLPE under the influence of high electrical field stress at a constant operating temperature or under an accelerating aging test. However, it was previously found that the IPM is not appropriate when XLPE is subjected to both low electrical and thermal stresses, which is similar to the actual conditions of power cables installed in electrical power transmission systems. From the experimental results of the lifetime of XLPE under electrical field stresses at specific temperatures, an inflection point was found at a certain electrical field stress $\left(E_{d}\right)$. From this observation and based on a physical interpretation, an engineering lifetime model was derived by separation of the lifetime curve into two parts, i.e., for electrical field stresses of lower than $E_{d}$ and higher than $E_{d}$. Nonlinear and linear least-squares methods were applied to fit the developed model with the experimental data, and the parameters of the developed engineering lifetime model were determined. With the developed model, the maximum electrical field stress at the maximum operating temperature can be determined, and it was utilized as a criterion in the design of power cables as an example in this study.

\section{Introduction}

Crosslinked polyethylene (XLPE) was invented almost half a century ago. ${ }^{(1)}$ It is used as the insulating material of power and telecommunication cables, which are called XLPE cables. This material is increasingly used since it has many advantages. For example, it can withstand temperatures of up to $90^{\circ} \mathrm{C}$ without deterioration and it has a long service lifetime.

The main cause of failure of XLPE power cables is the deterioration of the insulation, while a few percent of failures are caused by the conductor. Thus, the lifetime of XLPE cables can be predicted from the lifetime of XLPE. XLPE cables should have a lifetime of 40 to 60 years under normal operating conditions. ${ }^{(2,3)}$ 
The aging of XLPE cables is affected by electrical and thermal stresses. In practical operation, electrical field stress over the threshold electrical field stress $\left(E_{t h}\right)^{(4)}$ leads to deterioration of the insulation or aging according to an IEC standard. ${ }^{(5)}$ Hence, it is necessary to know the maximum electrical field stress at which XLPE cables can operate without aging of the insulation. Normally, $E_{t h}$ depends on the type of dielectric material and the temperature under operation.

There have been some studies related to lifetime models of XLPE. Some studies considered only the effect of thermal stress. ${ }^{(2,3,6)}$ The lifetime of XLPE can be estimated by investigation of the lifetime at high temperatures and then extrapolating the results to the operating temperature using the Arrhenius equation. ${ }^{(6)}$ The effect of only electrical field stress was investigated in other studies. The lifetime of XLPE used as power cable insulation was investigated under the influence of electrical field stress. The inversed power model (IPM) is commonly used to estimate the lifetime of dielectric materials ${ }^{(5,7-9)}$ because of its simple form. However, the model parameters have no significant physical meaning, and the IPM can only be applied in the range of high electrical field stresses for aging tests. Other studies considered the effect of combined electrical and thermal stresses. The lifetime of XLPE can be obtained by a combined stress model (CSM), as previously reported. ${ }^{(4,10-15)}$ This model was derived from the physical meaning of each parameter and has high accuracy. However, both the IPM and CSM require a large amount of experimental data for predicting the lifetime. This is inconvenient owing to the need for many experiments with long-time observation.

This paper presents an effective engineering model for estimating the lifetime of XLPE under the influence of electrical and thermal stresses. The proposed model requires only a small amount of experimental data, making it more practical in the field. On the basis of a derived formula with a physical interpretation, linear and nonlinear least-squares methods were applied to determine the model coefficients. This paper is organized as follows. Section 1 introduces the background and reviews the literature related to the lifetime model of XLPE under electrical and thermal stresses. Section 2 presents background theories of the conventional and proposed lifetime models. Section 3 presents our experimental setup for investigating the lifetime curves of XLPE. Section 4 presents an approach for parameter determination of the proposed lifetime model. Section 5 presents an application of the model to power cable design as an example. Finally, a conclusion is presented in Sect. 6.

\section{Conventional and Proposed Models}

\subsection{IPM}

When a dielectric is subjected to electrical field stress, the lifetime or time to dielectric breakdown is assumed to correspond to that obtained by the IPM, which is based on observation. ${ }^{(6)}$ The IPM is expressed as

$$
t=c E^{(-n)},
$$


where $t$ is the time to breakdown, $E$ is the electrical field stress, $c$ is a constant, and $n$ is the voltage endurance coefficient (VEC).

The plot of the material lifetime versus the time to breakdown on a log-log scale, called the voltage endurance line, yields a straight line as shown in Fig. 1. In this case, the VEC is the negative inverse of the slope of the straight line.

In general, the voltage endurance line of XLPE cables based on the previous literature ${ }^{(5,7)}$ is fitted with a straight line on a $\log -\log$ scale. However, in a real experiment, the lifetime characteristic of XLPE can be represented well with a concave upward and concave downward line as shown in Fig. 2. This means that the lifetime characteristic of XLPE can separated into two regions. One is the low-electrical-field region and the other is the high-electrical-field region. In this case, the VEC must be defined as the negative inverse of the slope as expressed by Eq. (2).

$$
-\frac{1}{V E C}=\frac{d \ln (E)}{d \ln (t)}
$$

Also, in the low-electrical-field stress range, the line approaches the threshold electrical field stress $\left(E_{t h}\right)$, below which there is no aging of the dielectric material. Hence, the IPM in Eq. (1) can be modified and expressed as Eq. (3), where $n$ is the VEC.

$$
t=c\left(\frac{E}{E_{t h}}\right)^{-n}
$$

\subsection{Arrhenius equation}

Considering XLPE under electrical field stress, energy exists in the form of heat transfer from the conductor to the insulator. This is directly related to the internal chemical reaction rate. ${ }^{(7)}$ The lifetime of a dielectric material depends on its chemical reaction rate. A higher

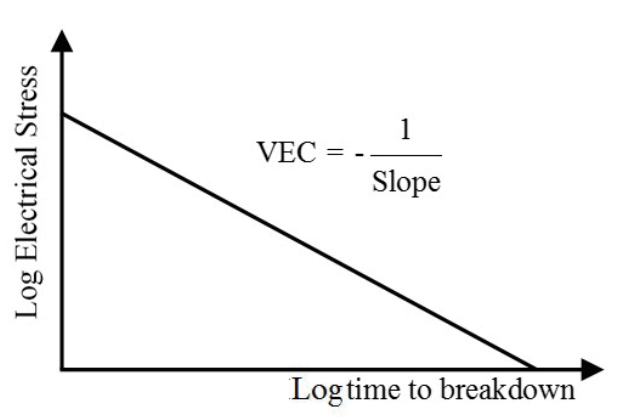

Fig. 1. Voltage endurance plotted on log-log graph.

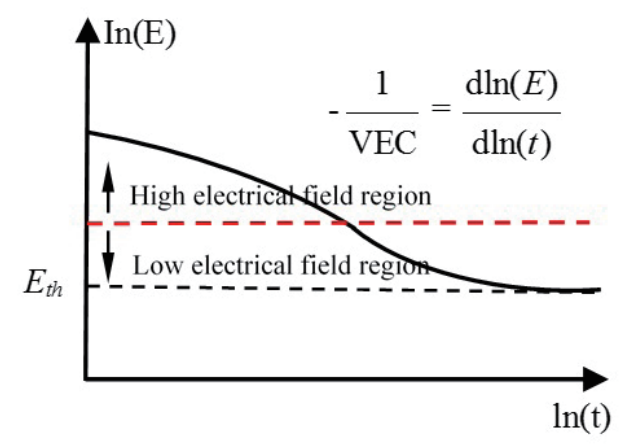

Fig. 2. (Color online) General lifetime curve of XLPE under electrical threshold stress. 
chemical reaction rate leads to a higher deterioration rate or a shorter lifetime. According to the transition state theory, ${ }^{(10)}$ the formation of products from reactants requires an energy at least equal to the activation energy $(\Delta G)$ as shown in Fig. 3. Hence, temperature and $\Delta G$ are major parameters impacting the chemical reaction rate. Under no electrical field stress, $\Delta G$ is high and there is no aging of the material.

In the Arrhenius equation given by Eq. (4), the chemical reaction rate $(R)$ is represented as a function of activation energy $(\Delta G)$ and temperature $(T)$.

$$
R=\frac{k_{B} T}{h} \exp \left(-\frac{\Delta G}{k_{B} T}\right)
$$

Here, $R$ is the chemical reaction rate, $k_{B}$ is Boltzmann's constant $\left(1.381 \times 10^{-23} \mathrm{~J} / \mathrm{K}\right), T$ is the temperature $(\mathrm{K})$, and $h$ is Planck's constant $\left(6.626 \times 10^{-34} \mathrm{Js}\right)$.

\subsection{Physical meaning of the VEC}

According to previous studies, ${ }^{(4,9,15)}$ the electrical threshold stress $\left(E_{t h}\right)$ affects the lifetime of XLPE used as the insulation of power cables. The chemical reaction rate $(R)$ is assumed to have a linear relationship with the lifetime $(t)$. From Eq. (4), $R$ can be rewritten as

$$
R=R_{0} \exp \left(n \ln \left(\frac{E}{E_{t h}}\right)\right),
$$

where $R_{0}$ is the initial chemical reaction rate and $n$ is the VEC.

When electrical field stress is applied to a material, its free energy is increased from $G_{1}$ to $G_{1}{ }^{\prime}$. This decreases the energy barrier and increases the reaction rate. By writing $\Delta W$ as the decrease in the barrier energy, Eq. (4) can be rewritten as Eq. (6), and the initial reaction rate can be written as Eq. (7).

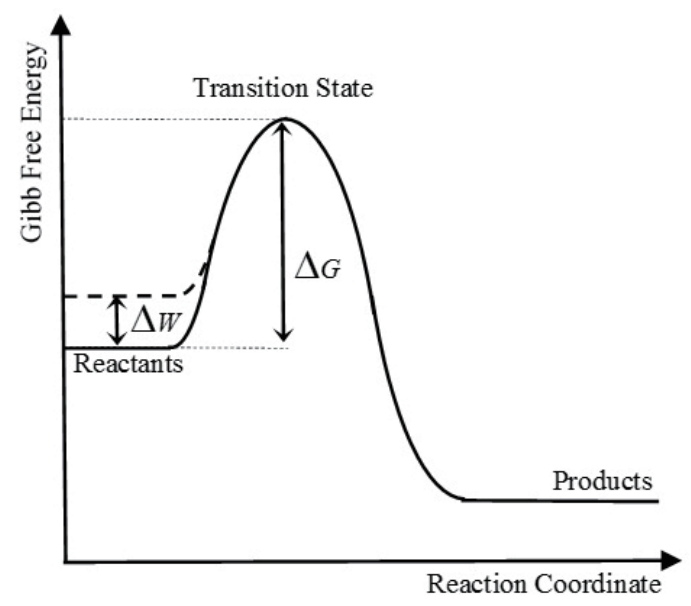

Fig. 3. Reaction coordinate diagram. 


$$
\begin{gathered}
R^{\prime}=\frac{k_{B} T}{h} \exp \left(-\frac{\Delta G-\Delta W}{k_{B} T}\right) \\
R_{0}=\frac{k_{B} T}{h} \exp \left(-\frac{\Delta G-\Delta W_{R=R_{0}}}{k_{B} T}\right)
\end{gathered}
$$

Substituting Eqs. (6) and (7) into Eq. (5) gives Eq. (8) as an expression for the VEC (n). Because $\Delta W_{R=R_{0}}$ is very small compared with $\Delta W$, the VEC ( $n$ ) can be written as Eq. (9).

$$
\begin{gathered}
n=-\frac{\Delta W-\Delta W_{R=R_{0}}}{k_{B} T \ln \left(\frac{E}{E_{t h}}\right)} \\
n=\frac{-\Delta W}{k_{B} T \ln \left(\frac{E}{E_{t h}}\right)}
\end{gathered}
$$

It is considered that XLPE aging is caused by electrons accelerated by electrical field stress through sub-microvoids (SMVs), resulting in damage to the polymer chains. ${ }^{(11,12)}$ The SMV size will suddenly increase when XLPE cables are subjected to high electrical field stress $\left(E>E_{d}\right)$. In this case, $\Delta W$ is proportional to temperature as follows:

$$
\Delta W=k\left(E-E_{d}\right)+\Delta W_{0}: E>E_{d},
$$

where $k$ is the constant of proportionality of $\Delta W$, and $E_{d}$ is the maximum electrical field stress before $\Delta W$ becomes proportional to the electrical field stress.

The SMV size is extremely small when XLPE cables are subjected to low electrical field stress $\left(E_{t h}<E \leq E_{d}\right)$. In this case, $\Delta W$ does not depend on the electrical field stress, i.e.,

$$
\Delta W=\Delta W_{0}: E_{t h}<E \leq E_{d},
$$

where $\Delta W_{0}$ is a constant.

\subsection{Proposed lifetime estimation model}

In the proposed engineering model, experimental data were employed to construct a model based on mathematical formulas, which were derived from a physical interpretation, increasing the practicality of the model in the field. On the basis of the derived formula, linear and nonlinear least-squares methods were applied to determine the model coefficients. The proposed model was derived as follows. 
Considering XLPE subjected to electrical field stress in the range of $E_{t h}<E \leq E_{d}$, the lifetime can be obtained by substituting $\Delta W$ in Eq. (11) into the VEC in Eq. (9), and substituting the VEC into the IPM in Eq. (2). The resulting equation is

$$
\frac{d \ln (E)}{d \ln (t)}=-\frac{k_{B} T \ln \left(\frac{E}{E_{t h}}\right)}{\Delta W_{0}} .
$$

The lifetime $(t)$ as a function of electrical field stress and temperature can be obtained by integration of Eq. (12), resulting in Eqs. (13) and (14), where $K$ is a constant of integration.

$$
\begin{gathered}
\int \frac{1}{n\left(E / E_{t h}\right)} d \ln (E)=-\int\left(\frac{k_{B} T l}{\Delta W_{0}}\right) d \ln (t) \\
\ln \left(\ln \left(\frac{E}{E_{t h}}\right)\right)=-\frac{k_{B} T}{\Delta W_{0}}\left(\ln \left(\frac{t}{t_{0}}\right)\right)+K
\end{gathered}
$$

By rearranging Eq. (14), the lifetime can be expressed as Eq. (15), then simplified to Eq. (16).

$$
\begin{gathered}
t=\left(\frac{t_{0}}{e^{K}}\right)\left(\ln \left(\frac{E}{E_{t h}}\right)\right)^{-\frac{\Delta W_{0}}{k_{B} T}} \\
t=c^{\prime}\left(\ln \left(\frac{E}{E_{t h}}\right)\right)^{-\frac{\Delta W_{0}}{k_{B} T}}
\end{gathered}
$$

Here, the coefficient $\left(c^{\prime}\right)$ is defined as $c^{\prime}=\left(t_{0} / e^{K}\right)$.

Considering XLPE subjected to electrical field stress in the range of $E>E_{d}$, the lifetime is a function of electrical field stress and temperature. It can be obtained by substituting $\Delta W$ in Eq. (10) into the VEC in Eq. (9) and substituting the VEC into Eq. (3). The resulting equation is Eq. (17), which can be simplified to Eqs. (18) and (19).

$$
\begin{gathered}
t=c(e)^{-\frac{k\left(E-E_{d}\right)+\Delta W_{0}}{K_{B} T}} \\
t=\left(c(e)^{\frac{k E_{d}-\Delta W_{0}}{K_{B} T}}\right)(e)^{-\frac{k E}{K_{B} T}}
\end{gathered}
$$




$$
t=c^{\prime \prime}\left(e^{-\frac{k E}{K_{B} T}}\right)
$$

Here, the coefficient $\left(c^{\prime \prime}\right)$ is defined as $c^{\prime \prime}=c(e)^{\frac{k E_{d}-\Delta W_{0}}{K_{B} T}}$.

\section{Experimental Setup}

In the experiment, the XLPE lifetime was investigated in accordance with the previous literature. ${ }^{(13,14)}$ The influence of electrical field stress at temperatures of 20,60 , and $90{ }^{\circ} \mathrm{C}$ was examined since the maximum operating temperature of the XLPE cables is approximately $90{ }^{\circ} \mathrm{C}$. The cross-sectional dimensions of a cable with $20 \mathrm{~cm}$ length are shown in Fig. 4 . The insulation thickness is $3.0 \mathrm{~mm}$, the shielding thickness is $1.0 \mathrm{~mm}$, and the conductor diameter is $1.8 \mathrm{~mm}$.

The test setup is shown in Fig. 5. The electrical field stress at both ends of the cable was controlled by conical grading rings (deflection conoids) made of Teflon, whose permittivity is almost the same as that of XLPE insulation. The conductor was subjected to a high voltage to obtain the required electrical field stress, while the XLPE insulation layer was covered by a thin sheet conductor connected to ground. The conductor was installed in a temperature-controlled oven.

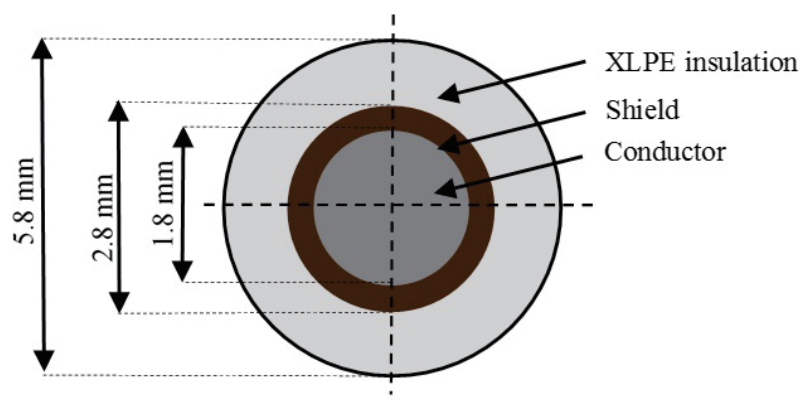

Fig. 4. (Color online) Cross-sectional dimensions of the investigated cable.

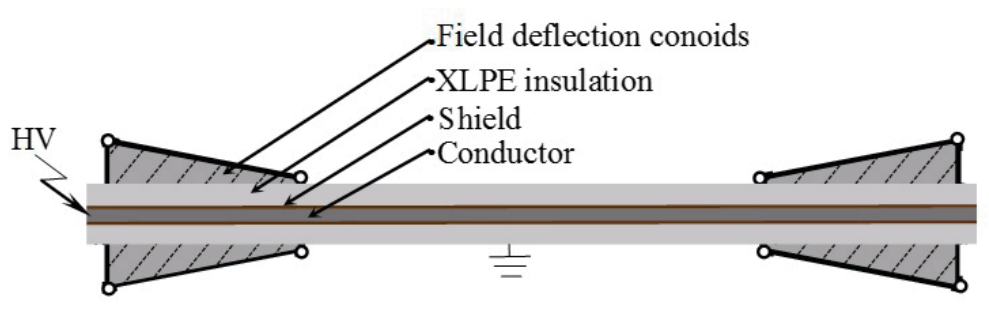

Fig. 5. (Color online) Test setup. 
The voltage was increased at a rate of $30 \mathrm{kV} / \mathrm{min}$. The tests were performed at temperatures of 20,60 , and $90^{\circ} \mathrm{C}$. The experimental results are shown in Fig. 6. Circles represent the results of the test performed at a frequency of $50 \mathrm{~Hz}$, whereas triangles represent the results of the test performed at a frequency of $450 \mathrm{~Hz}$. Also, the rhombus represents data obtained from a combined analysis method. ${ }^{(14)}$

From the experimental results of the XLPE lifetime under electrical field stresses at specific temperatures in Fig. 6, an inflection point was found at a certain electrical field stress $\left(E_{d}\right)$. It is clear that the lifetime curve should be divided into two parts, which is consistent with the proposed model.

\section{Parameter Determination of the Proposed Lifetime Model}

To determine the parameters of the proposed lifetime model, we first found the coefficients or the parameters of the lifetime curve of the XLPE under the various electrical field stresses at the specific temperatures of 20,60 , and $90{ }^{\circ} \mathrm{C}$. Nonlinear regression based on the Levenberg-Marquardt algorithm was applied to fit the lifetime data at each temperature, and the parameters in Eqs. (16) and (19) were determined at the temperature. The results of the fitting are shown in Fig. 7 and are in good agreement with the experimental data. The R-squared values $\left(R^{2}\right)$ of the results for the temperatures of 20,60 , and $90{ }^{\circ} \mathrm{C}$ are $0.9970,0.9979$, and 0.9994 , respectively. Note that the determined parameters $\left(E_{t h}, \Delta W_{0}, C^{\prime}, k, C^{\prime \prime}\right)$ in Table 1 are proportional to the temperature.

Then, linear regression was applied to fit the determined parameters at the three different operating temperatures, and the regression results are expressed as follows:

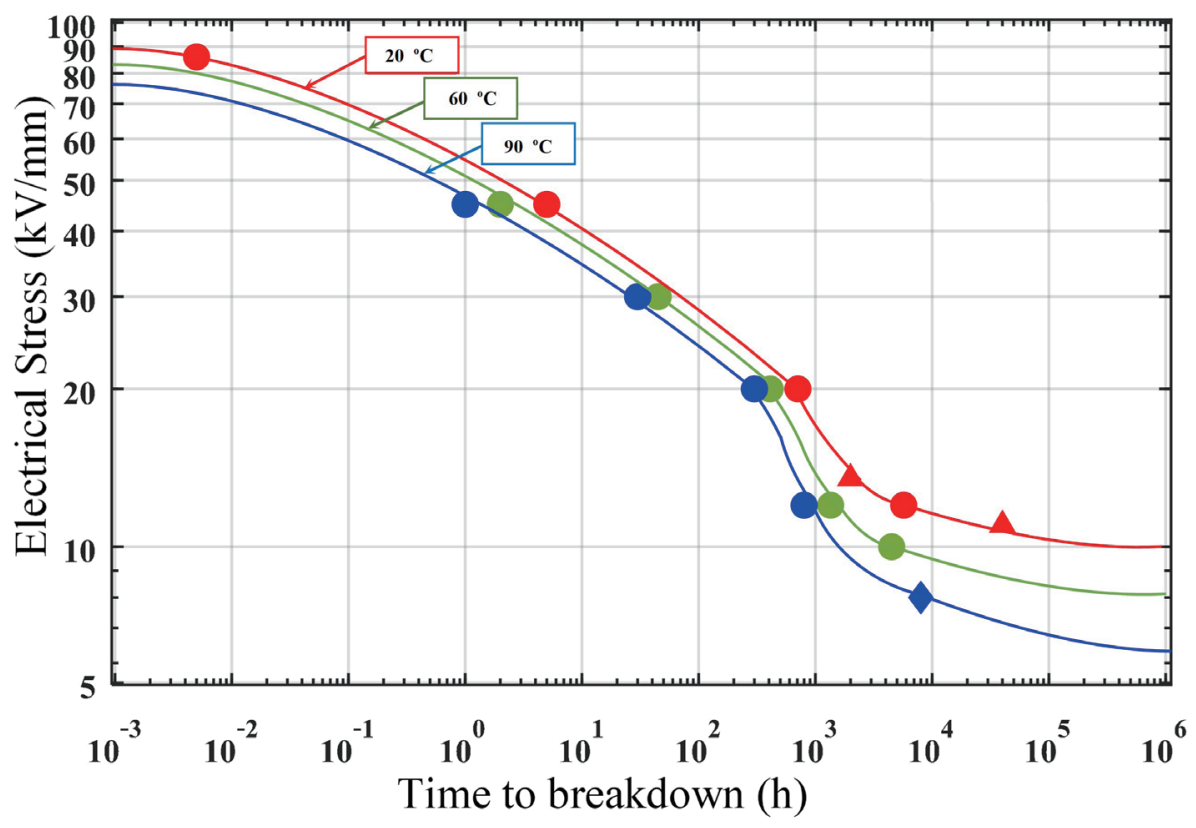

Fig. 6. (Color online) XLPE lifetime curves at temperatures of 20, 60, and $90^{\circ} \mathrm{C}$. 


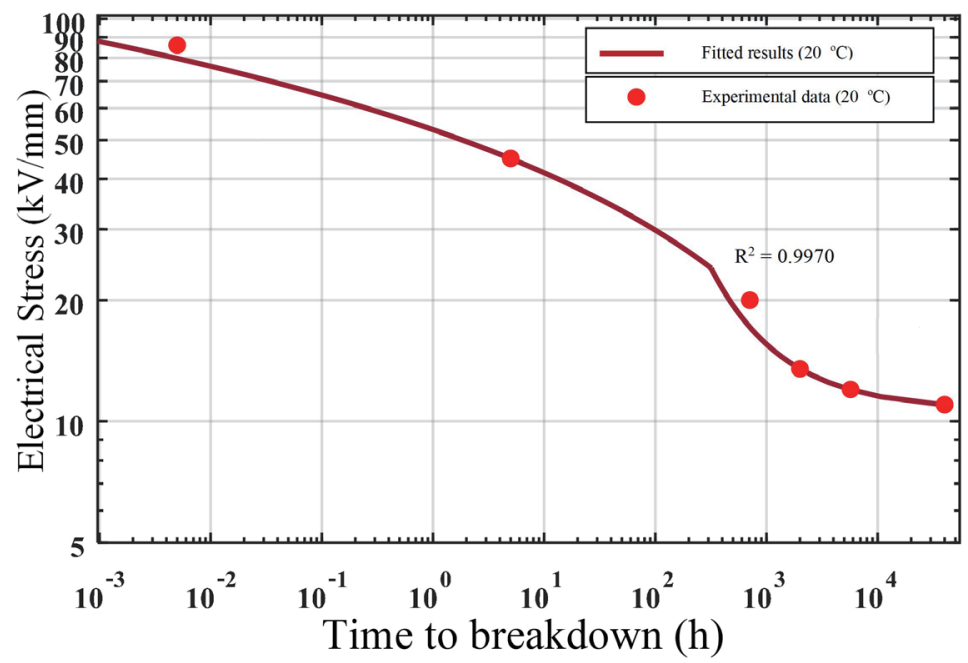

(a)

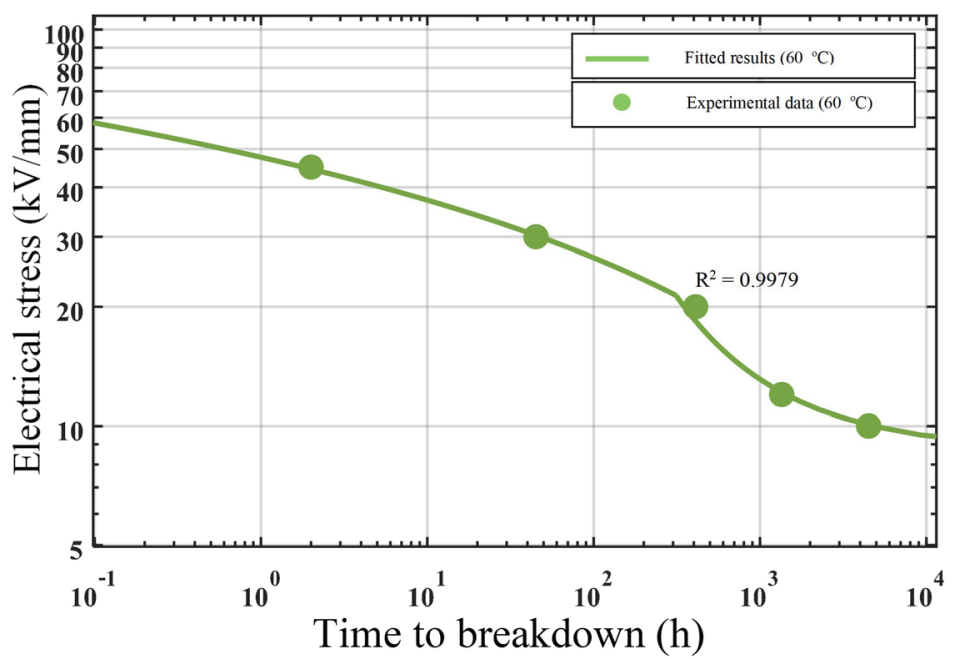

(b)

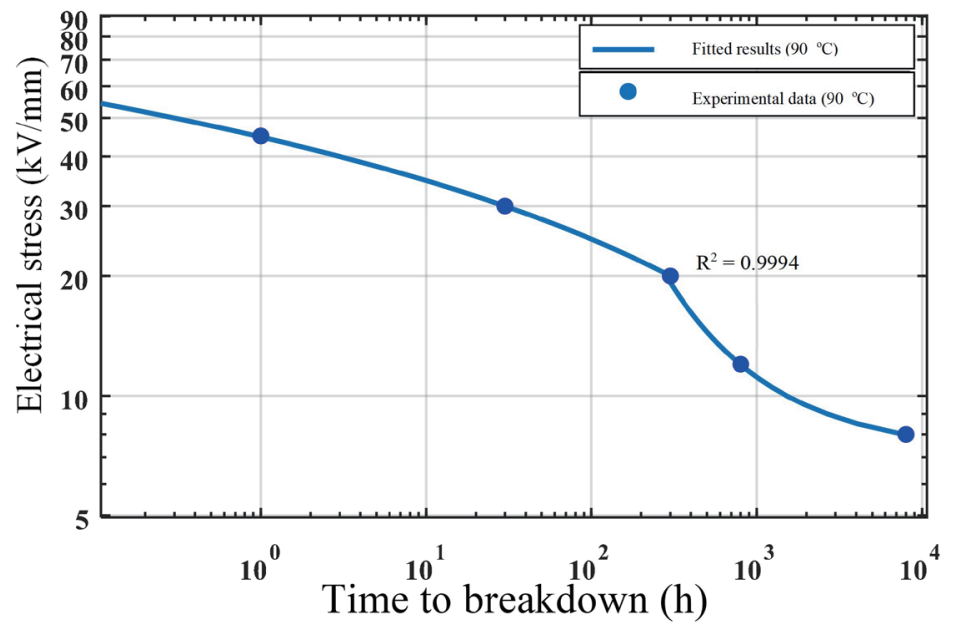

(c)

Fig. 7. (Color online) Experimental data (circles) in comparison with the fitting results. 
Table 1

Fitted parameters at temperatures of 20,60 , and $90{ }^{\circ} \mathrm{C}$.

\begin{tabular}{lccc}
\hline \multirow{2}{*}{ Parameter } & \multicolumn{3}{c}{ Temperature } \\
\cline { 2 - 4 } & $20^{\circ} \mathrm{C}$ & $60{ }^{\circ} \mathrm{C}$ & $90{ }^{\circ} \mathrm{C}$ \\
\hline$E_{t h}$ & 10.65 & 8.594 & 7.153 \\
$\Delta W_{0}$ & $6.07\left(10^{-21}\right)$ & $6.98\left(10^{-21}\right)$ & $7.51\left(10^{-21}\right)$ \\
$C^{\prime}$ & 235.3 & 265.4 & 299.7 \\
$k$ & $8.01\left(10^{-22}\right)$ & $1.02\left(10^{-21}\right)$ & $1.15\left(10^{-21}\right)$ \\
$C^{\prime \prime}$ & $3.72\left(10^{4}\right)$ & $3.42\left(10^{4}\right)$ & $3.00\left(10^{4}\right)$ \\
\hline
\end{tabular}

$$
\begin{gathered}
E_{t h}=-0.05 T+25.29, \\
\Delta W_{0}=2.07\left(10^{-23}\right) T, \\
c^{\prime}=0.91 T-33.5, \\
k=5.048\left(10^{-24}\right) T-6.745\left(10^{-22}\right), \\
c^{\prime \prime}=-102.2 T+67440,
\end{gathered}
$$

where $T$ is temperature in the unit of $\mathrm{K}$.

According to the experimental data in Fig. 6, the inflection points of the electrical field stresses $\left(E_{d}\right)$ are approximately in the range of 15 to $30 \mathrm{kV} / \mathrm{mm}$. Also, it was found that $E_{d}$ is proportional to temperature. Thus, $E_{d}$ can be written as a function of temperature and can be fitted by linear regression as follows:

$$
E_{d}=-0.055 T+39.82
$$

From all above results in Eqs. (16), (19), and (20)-(25), the lifetime of the considered XLPE under electrical field and thermal stresses can be plotted as the contours shown in Fig. 8.

\section{Application in Cable Insulation Design}

The data of XLPE lifetime under the influence of electrical and thermal stresses are necessary for power cable design. Therefore, many experiments with lengthy observations are required to obtain such data. However, the proposed model utilizes the appropriate formula and curve fitting method to estimate the lifetime, thus requiring only a small amount of experimental data and making it more practical for applications in the field.

In the insulation design of power cables, it is necessary to know the maximum electrical field stress at the maximum operating temperature that the insulation can endure without deterioration. In the case of XLPE, the maximum operating temperature is considered to be $90{ }^{\circ} \mathrm{C}$. The maximum electrical field stress used in power cable design depends on the power system voltage. For distribution systems (with a system voltage of up to $36 \mathrm{kV}$ ), a maximum 

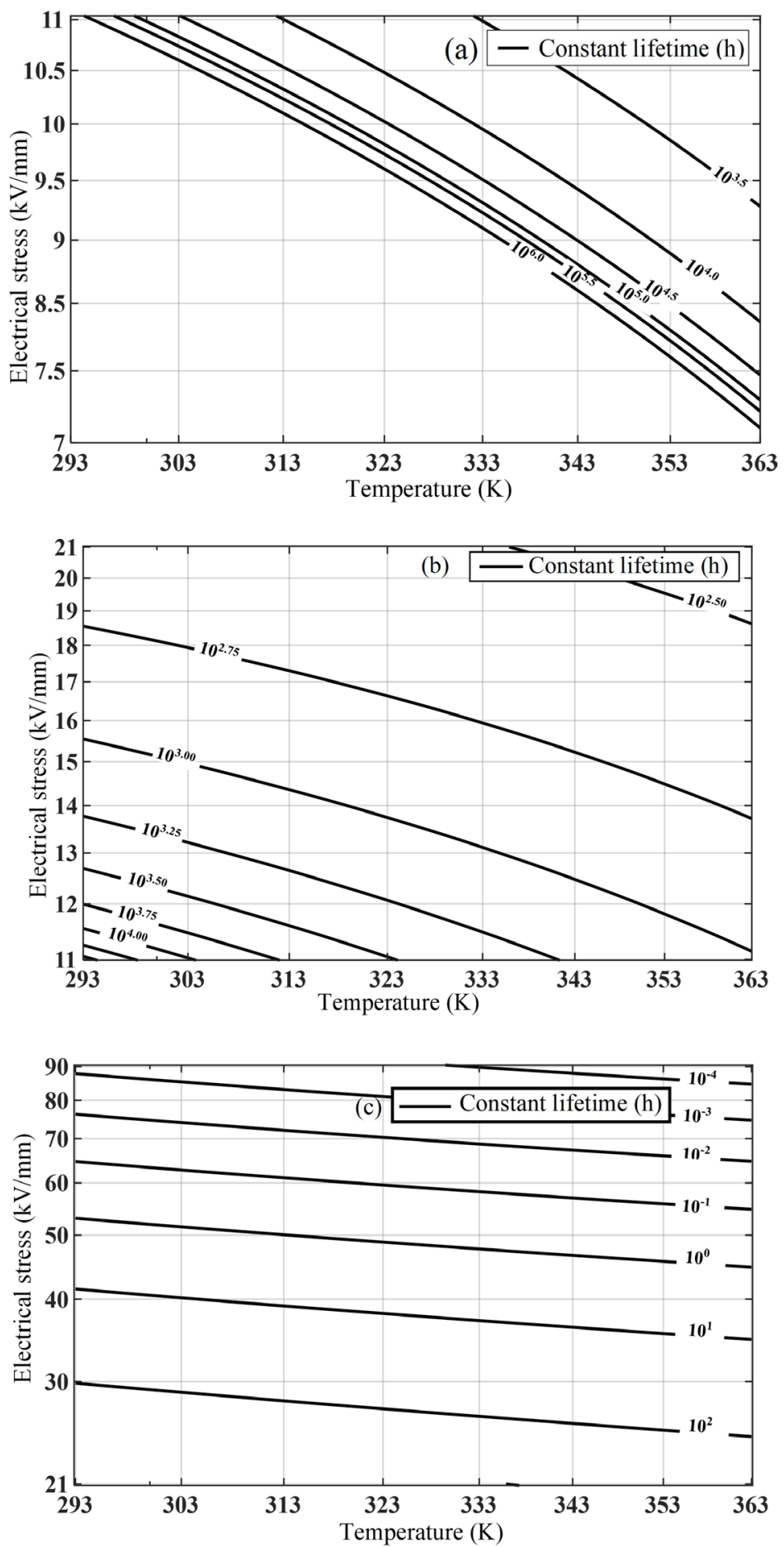

Fig. 8. Lifetime of the considered XLPE under electrical field and thermal stresses.

electrical field stress of about $5 \mathrm{kV} / \mathrm{mm}$ is used for power cable design. For higher-voltage systems, the designed maximum electrical field stress may reach the order of $10 \mathrm{kV} / \mathrm{mm}$, and an additive to prevent water treeing must be added to the XLPE used in the power cable. ${ }^{(15)}$ In this paper, XLPE without such an additive was used in the model development. 
The maximum $E_{t h}$ occurring at the maximum operating temperature of $90{ }^{\circ} \mathrm{C}$ was calculated to be $7.13 \mathrm{kV} / \mathrm{mm}$ from Eq. (20). Therefore, the designed maximum electrical field stress in the power cable must be less than this value. For better understanding, the insulation design of a power cable was considered. The voltage rating of this power cable was $36 / 69(72.5 \mathrm{kV})$, and the cross-sectional area of the conductor was $185 \mathrm{~mm}^{2}$, corresponding to an outer diameter of the conductor of $15.8 \mathrm{~mm}$. With the XLPE considered in Sect. 3, the insulation thickness $(d)$ of this cable can be calculated using

$$
\begin{gathered}
E_{\max }=\frac{U_{p}}{r_{1} \ln \left(r_{2} / r_{1}\right)}, \\
d=r_{2}-r_{1},
\end{gathered}
$$

where $r_{1}$ and $r_{2}$ are the inner and outer radii of the insulation, respectively, and $U_{p}$ is the peak voltage across the insulation.

In this case, $E_{\max }=7.13 \mathrm{kV} / \mathrm{mm}, r_{1}=7.9 \mathrm{~mm}$, and $U_{p}=59.19 \mathrm{kV}$. Therefore, $r_{2}=22.59 \mathrm{~mm}$ and $d=14.69 \mathrm{~mm}$. To ensure safety, the insulation thickness should be at least $15 \mathrm{~mm}$.

\section{Conclusion}

We have presented an effective approach for determining the lifetime of XLPE under electrical and thermal stresses. The considered electrical field and operating temperature were in the ranges of 7 to $90 \mathrm{kV} / \mathrm{mm}$ and 20 to $90{ }^{\circ} \mathrm{C}$, respectively. From the experimental results of the XLPE lifetime under electrical field stresses at specific temperatures, an inflection point was found at a certain electrical field stress $\left(E_{d}\right)$. From this observation and on the basis of a physical interpretation, an engineering lifetime model was derived by separating the lifetime curve into two parts, i.e., at electrical field stresses below $E_{d}$ and above $E_{d}$. Nonlinear and linear regression methods were applied to fit the developed model with the experimental data, and the parameters of the developed engineering lifetime model were determined. It was found that the proposed model fitted the experimental data very well, and the $R^{2}$ value was very close to one. With the developed model, the maximum electrical field stress (the threshold electrical field stress, $E_{t h}$ ) at the maximum operating temperature can be determined, which was utilized as a criterion in the design of power cables as an example. It can be concluded that the proposed lifetime model and the presented approach for parameter determination are very attractive for the lifetime estimation of XLPE under electrical and thermal stresses and for power cable design.

\section{Acknowledgments}

The authors would like to give their special acknowledgment to the School of Engineering, King Mongkut's Institute of Technology Ladkrabang, for providing the facility in this research work. This project was funded by the National Research Council of Thailand. 


\section{References}

1 H. Orton: IEEE Trans. Dielectr. Electr. Insul. 29 (2013) 52. https://doi.org/10.1109/MEI.2013.6545260

2 A. S. Alghamdi and R. K. Desuqi: Heliyon 6 (2020). https://doi.org/10.1016/j.heliyon.2019.e03120

3 Z. Zhang, P. Dieu, S. Assala, and L. Wu: Electr. Power Syst. Res. 163 (2018) 572. https://doi.org/10.1016/j. epsr.2017.12.027

4 G. Bahder, T. Garrity, M. Sosnowski, R. Eaton, and C. Katz: IEEE Trans. Power Apparatus Syst. PAS-101 (1982) 1379. https://doi.org/10.1109/TPAS.1982.317185

5 Electricalinsulating Materials and Systems - AC Voltage Endurance Evaluation: International Standard IEC 61251, 1st ed. (Geneva, Switzerland, 2015).

6 C. Dang, J.-L. Parpal, and J.-P. Crine: IEEE Trans. Dielectr. Electr. Insul. 3 (1996) 237. https://doi. org/10.1109/94.486776

7 M. Cacciari, G. C. Montanari, L. Simoni, A. Cavallini, and A. Motori: Proc. 1991 IEEE Power Engineering Society Transmission and Distribution Conf. (2002).

8 E. V. Anslyn and D. A. Dougherty: Modern Physical Organic Chemistry (University Science Books Wiley, 1960) Chap. 6.

9 H. S. Endicott, B. Hatch, and R. Sohmer: IEEE Trans. Compon. Parts 12 (1965) 34. https://doi.org/10.1109/ TCP.1965.1135088

10 G.C.Momtanary: IEEE Trans. Electr. Insul. 27 (1992) 974. https://doi.org/10.1109/TEI.1992.4783194

11 H. Bien, L. Yang, Z. Ma, B. Deng, H. Zhang, and Z. Wu: IEEE Trans. Dielectr. Electr. Insul. 27 (2020) 132. https://doi.org/10.1109/TDEI.2019.008351

12 G. C. Montanari, G. Pattini, and L. Simoni: IEEE Trans. Power Delivery 2 (1987) 596. https://doi.org/10.1109/ TPWRD.1987.4308151

13 L. Simoni: IEEE Trans. Electr. Insulation EI-16 (1981) 277. https://doi.org/10.1109/TEI.1981.298361

14 Y. Namiki, H. Shimanuki, F. Aida, and M. Morita: IEEE Trans. Electr. Insul. EI-15 (1980) 473. https://doi. org/10.1109/TEI.1980.298276

15 T. Worzyk: Submarine Power Cables (Springer, Heidelberg, 2005) p. 21.

\section{About the Authors}

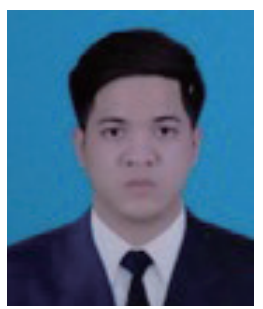

Sarawuth Pramualsingha received his B.Eng. degree from King Mongkut's Institute of Technology Ladkrabang (KMITL), Thailand, in 2020. Since 2020, he has been at KMITL, where he is an M.Eng. student in the Department of Electrical Engineering. His research interests are in high-voltage engineering. (63601077@kmitl.ac.th)

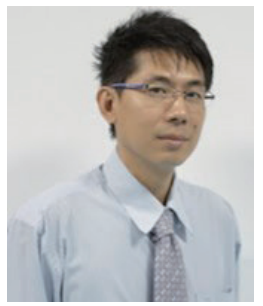

Peerawut Yutthagowith received his B.Eng. and M.Eng. degrees from Chulalongkorn University, Thailand, in 1998 and 2001, respectively, and his Ph.D. degree from Doshisha University, Japan, in 2010. Since 2007, he has been at King Mongkut's Institute of Technology Ladkrabang. His research interests are in high-voltage engineering applications and sensors.

(peerawut.yu@kmitl.ac.th) 